\title{
On the Detectability of Gamma Rays from Clusters of Galaxies: Mergers versus Secondary Infall
}

\author{
Stefano Gabici ${ }^{a, 1}$ Pasquale Blasi ${ }^{\text {b,c, }, 2}$ \\ ${ }^{a}$ Dipartimento di Astronomia, Università di Firenze, \\ Largo E. Fermi, 5 - I50125 Firenze, Italy \\ ${ }^{\mathrm{b}}$ INAF/Osservatorio Astrofisico di Arcetri \\ Largo E. Fermi, 5 - I50125 Firenze, Italy \\ ${ }^{\mathrm{c}}$ INFN, Sezione di Firenze, Italy
}

\begin{abstract}
Particle acceleration in clusters of galaxies is expected to take place at both merger and accretion shocks. The electron component may be energized to energies of several $\mathrm{TeV}$, and upscatter a small fraction of the photons in the cosmic microwave background up to gamma ray energies. We address here the issue of the detectability of the gamma radiation generated either in merger events or during the accretion of matter onto cluster potential wells. The predictions are specialized to the cases of GLAST and AGILE, for which a few tens of clusters are expected to be detected, and to Cherenkov telescopes, for which however the perspectives do not appear to be optimistic.
\end{abstract}

\section{Introduction}

Observations of radio [1] and hard X-ray [2] emission from several clusters of galaxies promoted these objects to the role of largest nonthermal sources in the universe. On the theoretical side, two ideas contributed to reinforce the belief that clusters of galaxies can be interesting as high energy radiation emitters: in $[3,4]$ it was first understood that cosmic rays accelerated within the cluster volume would be confined there for cosmological time scales, enhancing the possibility of inelastic proton-proton collisions and consequent gamma ray

1 E-mail: gabici@arcetri.astro.it

2 E-mail: blasi@arcetri.astro.it

Preprint submitted to Elsevier Science 2 November 2018 
production through the decay of neutral pions. In [5] it was recognized that large scale shocks associated with the formation of structures in the universe, may accelerate electrons to $\mathrm{TeV}$ energies, implying that high energy emission would occur due to the upscatter of the photons of the cosmic microwave background $(\mathrm{CMB})$ to gamma ray energies, through inverse Compton scattering (ICS).

The possibility that single clusters could be sources of high energy gamma rays fueled much interest also in the related possibility that clusters could in fact be also responsible for the observed isotropic diffuse gamma ray background (DGRB) [6], thought to be of extragalactic origin (although the possibility that at least a fraction of this background might in fact be explained in terms of ICS from electrons in the halo of our Galaxy [7]). In [8] it was found that the diffuse background due to inelastic interactions of cosmic rays trapped in the intracluster medium could not add up to more than $2 \%$ of the observed flux above $100 \mathrm{MeV}$, confirming previous calculations illustrated in [3]. The acceleration of electrons at the shocks related to the process of structure formation was more recently considered in [5], where the observed DGRB was found to be saturated by the cluster contribution. A more realistic calculation [9] showed that ICS of electrons in clusters cannot contribute more than $\sim 10 \%$ of the observed DGRB (of this, only $\sim 1 \%$ is actually related to cluster mergers, while most of the flux comes from electrons accelerated at accretion shocks). This result is in closer agreement with the results obtained in recent numerical calculations $[10,11]$.

Although it seems now unlikely that clusters may play an important role in explaining the bulk of the extragalactic DGRB, it seems plausible that future gamma ray telescopes, especially in space, may finally be able to detect the gamma ray glow associated with the formation of single clusters of galaxies. In this paper we analyze this possibility more quantitatively, making use of a formalism developed in $[12,9]$ that allows us to evaluate the strength of the shocks that develop during mergers of two clusters, or during accretion of new matter onto an already existing cluster potential well. The important point made in [12] is that most merger related shocks are weak and unable to accelerate particles efficiently, a result later confirmed in [13] and in numerical simulations [14].

Our calculations show that a gamma ray telescope like GLAST should be able to detect $\sim 50$ clusters of galaxies at energies above $100 \mathrm{MeV}$. It is interesting that several clusters are expected to be detected even with AGILE, while we confirm the undetectability of these sources with EGRET. This is consistent with the recent results in [15] where new upper limits on the gamma ray emission from Abell clusters have been found, using old EGRET data.

The situation looks much less promising for ground based Cherenkov experi- 
ments, which are optimized for point sources: nearby clusters, with larger fluences, appear as relatively extended sources for these experiments, so that the corresponding potential for detection is lowered. Clusters at larger distances are point sources, but their fluence is well below the detection threshold of Cherenkov experiments. Moreover, at energies in excess of a few hundred GeV the effect of absorption in the cosmological infrared background becomes important, and contributes to further suppress the flux of gamma radiation from distant clusters. It is therefore unlikely that clusters of galaxies may be seen as shining at $100-1000 \mathrm{GeV}$ energies in the near future.

The paper is planned as follows: in $\S 2$ we describe the acceleration of particles at shocks occurring during structure formation. In $\S 3$ we summarize our recipe for the calculation of the strength of the shocks associated with the process of structure formation. The consequences on the spectra of shock accelerated particles are also summarized. In $\S 4$ we assess the detectability of clusters of galaxies with space-borne experiments, specializing our predictions to the upcoming AGILE and GLAST satellites. In $\S 5$ the issue of detectability is discussed in connection with ground based Cherenkov experiments. We conclude in $\S 6$.

\section{Shock acceleration during structure formation}

The hot gas in the intracluster medium is the proof that structure formation proceeds through shock waves, that heat up the gas to the virial temperature whose value is determined by the depth of the dark matter potential well. The conversion of gravitational energy of the dark matter into thermal energy of the gas component occurs when the gas crosses shock waves that are created during the process of structure formation.

Diffusive acceleration can take place at these shocks, so that a small fraction of the particles in the accreting gas is extracted from the thermal distribution and energized to nonthermal energies. The acceleration time, necessary to energize a particle to energy $E$ is given by [16]:

$$
\tau_{a c c}(E)=\frac{3}{u_{1}-u_{2}}\left[\frac{D_{1}(E)}{u_{1}}+\frac{D_{2}(E)}{u_{2}}\right],
$$

where $u_{1}$ and $D_{1}(E)\left(u_{2}\right.$ and $\left.D_{2}(E)\right)$ are the fluid speed and diffusion coefficient of the particles upstream (downstream). From the physical point of view, $\tau_{a c c}$ is the sum of the residence times of particles upstream (index 1) and downstream (index 2). For well behaved diffusion coefficients, larger magnetic field strengths correspond to lower values of the diffusion coefficient, namely 
particles diffuse more slowly when the magnetic field is larger. This immediately suggests that the diffusion coefficient upstream is larger than the same quantity (at the same particle energy) downstream, so the residence time of the particles upstream is larger than the residence time in the downstream region. The acceleration time can therefore be approximated as follows:

$$
\tau_{a c c}(E) \approx \frac{3 D_{1}(E) r}{u_{1}^{2}(r-1)}=\frac{4 D_{1}(E)}{u_{1}^{2}}\left\{\frac{\mathcal{M}^{2}}{\mathcal{M}^{2}-1}\right\}
$$

where we introduced the compression factor $r=u_{1} / u_{2}$ and we used the relation between $r$ and the Mach number $\mathcal{M}, r=4 \mathcal{M}^{2} /\left(\mathcal{M}^{2}+3\right)$. It is worth noticing that the acceleration time depends in first approximation only on quantities that refer to the upstream fluid, where the magnetic field has not been affected yet by the passage of the shock, and preserves therefore its pre-shock structure. One may argue that the diffusion coefficient in the downstream region is dominated by the scattering of particles on magnetic field fluctuation lengths comparable to the Larmor radius of the particles, namely $D_{2}(E) \approx(1 / 3) c E / e B_{2}$. It is likely that these fluctuations are in fact created by the accelerated particles themselves. In the upstream region however it is possible that the diffusion coefficient is determined by the magnetic field structure pre-existing the shock transit. In principle the momentum and magnetic field dependence of the two fields upstream and downstream may be different.

For simplicity and convenience we assume here that the diffusion coefficient on both sides is Böhm-like. Moreover, since the term in brackets in Eq. 2 varies between 1.8 and 1 when the Mach number changes between 1.5 and infinity, we neglect this term for the purpose of estimating the acceleration time, therefore the acceleration time becomes:

$$
\tau_{a c c} \approx 0.3 B_{\mu}^{-1} E(G e V)\left(\frac{u_{1}}{10^{8} \mathrm{~cm} / \mathrm{s}}\right)^{-2} \text { years. }
$$

We stress again here that the magnetic field $B_{\mu}$ and velocity $u_{1}$ refer to the unshocked medium. Here we focus our attention on electrons. Their energy losses are dominated by ICS, with time scale:

$$
\tau_{I C S}=\frac{E}{\frac{4}{3} \sigma_{T} c U_{C M B}\left(\frac{E}{m_{e} c^{2}}\right)^{2}}=10^{9} E(G e V)^{-1} \text { years, }
$$

where $U_{C M B}$ is the energy density in the CMB radiation. Equating the acceleration and losses time scales we obtain an estimate of the maximum energy 
of accelerated electrons in case of Böhm diffusion:

$$
E_{\text {max }} \approx 57 B_{\mu}^{1 / 2}\left(\frac{u_{1}}{10^{8} \mathrm{~cm} / \mathrm{s}}\right) \mathrm{TeV}
$$

For electron energies up to $\sim 400 \mathrm{TeV}$ the ICS off the CMB photons occurs in the Thomson regime, therefore the maximum energy of the radiated photons is simply:

$$
E_{\gamma, \max }=\frac{4}{3}\left(\frac{E}{m_{e} c^{2}}\right)^{2} \epsilon_{C M B} \approx 7.5 T e V B_{\mu}\left(\frac{u_{1}}{10^{8} \mathrm{~cm} / \mathrm{s}}\right)^{2}
$$

As shown in $[12,9]$, if the diffusion coefficient in the unshocked medium is not Böhm-like, then the maximum energy of the electrons may be appreciably lower than found in Eq. (5), and insufficient to upscatter the CMB photons to gamma ray energies. In the following we assume that the maximum energy is well defined by Eq. (5).

The value of the magnetic field to adopt in our calculations depends crucially on whether we are describing particle acceleration at merger related shocks, or at accretion shocks. The former have relatively low Mach numbers [12] and occur in the virialized regions of clusters of galaxies, where the magnetic field is expected to be in the $\mu G$ range. Accretion shocks propagate in a cold unshocked medium, where the magnetic field is expected to set at the cosmological value, for which only upper limits are available. The field in the intracluster medium could be the result of magnetic pollution from sources within the cluster [17], being therefore unrelated to the magnetic field in the intergalactic medium outside clusters. Alternatively, the intracluster magnetic field could result from the compression of the external field, if the latter is of cosmological origin. The energy of the photons radiated by the accelerated electrons through ICS is above $E_{\gamma}$ whenever the magnetic field $B_{\mu}$ is larger than $10^{-9}\left(u_{1} / 1000 \mathrm{~km} / \mathrm{s}\right)^{-2}\left(E_{\gamma} / 10 \mathrm{GeV}\right)$ Gauss. This bound is certainly satisfied by the intracluster magnetic field, not necessarily by the poorly known field in the intergalactic medium outside clusters, for which upper limits of $10^{-9}-10^{-11}$ have been found, depending on the field structure [18]. On the other hand this constraint has to be taken with caution for the case of accretion shocks, because in a more realistic situation, as suggested by numerical simulations, high Mach number shocks are located close to filaments, where a substantial compression of cosmological fields might have occurred. We assume in the following that the electrons accelerated at both merger and accretion shocks are energetic enough to radiate gamma ray photons up to the $\sim T e V$ region.

In the next section we will show that there is a wide spread in the Mach numbers from about unity to $~ 1000$ [19], with a peak around 1.5 [12] for the 
weak merger related shocks. There is a correspondingly large spread in the power law index of the accelerated particles, while very little spread exists in the maximum energy that can be achieved at these shocks.

\section{Structure formation and strength of the merger and accretion shocks}

It is commonly believed that clusters of galaxies form hierarchically, with smaller dark matter halos merging together to form more massive halos. The shock surfaces that form during these mergers are responsible for the conversion of gravitational energy into thermal energy of the intracluster medium. Parallel to the merger processes, a secondary infall [20] of gas occurs at all times onto the potential well which is being formed. The information about the virialization of the inner region of this accretion flow is propagated outwards through a so-called accretion shock. While merger related shocks form and propagate in the virialized gas, accretion shocks propagate in the cold $\left(T \sim 10^{4}-10^{6} \mathrm{~K}\right)$ intergalactic medium, and therefore have, almost by definition, very large Mach numbers. In first approximation the position of the accretion shock is roughly coincident with the virial radius of the cluster.

In [9] we introduced a clear distinction between merger related shocks and accretion shocks, as seen from the perspective of nonthermal phenomena rather than from the point of view of structure formation. This classification however turns out to be useful for structure formation studies as well [14].

A useful analytical description of this hierarchical clustering was proposed by Press and Schechter (PS) [21] and subsequently developed, among others, by Lacey and Cole [22] in the form of the so-called extended Press-Schechter formalism. Within this approach, the comoving number density of clusters with mass $M$ at cosmic time $t$ is given by:

$$
\frac{d n(M, t)}{d M}=\sqrt{\frac{2}{\pi}} \frac{\varrho}{M^{2}} \frac{\delta_{c}(t)}{\sigma(M)}\left|\frac{d \ln \sigma(M)}{d \ln M}\right| \exp \left[-\frac{\delta_{c}^{2}(t)}{2 \sigma^{2}(M)}\right]
$$

while the rate at which clusters of mass $M$ merge at a given time to form clusters with final mass $M^{\prime}$ is:

$$
\begin{gathered}
\mathcal{R}\left(M, M^{\prime}, t\right) d M^{\prime}= \\
\sqrt{\frac{2}{\pi}}\left|\frac{d \delta_{c}(t)}{d t}\right| \frac{1}{\sigma^{2}\left(M^{\prime}\right)}\left|\frac{d \sigma\left(M^{\prime}\right)}{d M^{\prime}}\right|\left(1-\frac{\sigma^{2}\left(M^{\prime}\right)}{\sigma^{2}(M)}\right)^{-3 / 2}
\end{gathered}
$$




$$
\exp \left[-\frac{\delta_{\mathrm{c}}^{2}(\mathrm{t})}{2}\left(\frac{1}{\sigma^{2}\left(\mathrm{M}^{\prime}\right)}-\frac{1}{\sigma^{2}(\mathrm{M})}\right)\right] \mathrm{dM}^{\prime}
$$

Here $\varrho$ is the present mean density of the universe, $\delta_{c}(t)$ is the critical density contrast linearly extrapolated to the present time for a region that collapses at time $t$, and $\sigma(M)$ is the current rms density fluctuation smoothed over the mass scale $M$ (see [23,24] for more details).

As discussed above, shock waves form naturally in the baryonic component of clusters of galaxies involved in a merger, due to the supersonic motion of the two clusters. In [12] we developed an analytical recipe that allows us to estimate the Mach number of merger related shocks, once the masses of the two merging clusters and the redshift where the merger event occurs are given. The virial radii of the two clusters are written in the form

$$
R_{i}=\left(\frac{G M_{i}}{100 \Omega_{m} H_{0}^{2}\left(1+z_{f, i}\right)^{3}}\right)^{1 / 3},
$$

where $\Omega_{m}$ is the fraction of matter in the universe, compared to the critical density, $H_{0}$ is the Hubble constant and $z_{f, i}$ is the formation redshift of the $i$-th cluster. The relative velocity $v$ of the two merging clusters, with mass $M_{1}$ and $M_{2}$ respectively can be evaluated from energy conservation:

$$
-\frac{G M_{1} M_{2}}{R_{1}+R_{2}}+\frac{1}{2} M_{r} v_{r}^{2}=-\frac{G M_{1} M_{2}}{2 R_{t a}}
$$

where $M_{r}$ is the reduced mass, and $R_{t a}$ is the turnaround radius where the clusters are supposed to be at rest with respect to each other. In an EinsteinDe Sitter cosmology the turnaround radius is equal to twice the virial radius of the system and this result holds in approximate way also for a flat, lambda cosmology [25]. The virial radius depends on the cluster formation redshift, that we estimate following [22].

The sound speed in a cluster of mass $M_{i}$, needed in order to evaluate the Mach numbers, can be written as follows:

$$
c_{s, i}^{2}=\gamma(\gamma-1) \frac{G M_{i}}{2 R_{i}}
$$

where $\gamma=5 / 3$ is the adiabatic index of the gas.

Using this recipe, we can determine the probability of having a merger shock with a given Mach number, simply by simulating the merger tree of a cluster with given mass today. The cumulative probability distribution of shock Mach numbers, as obtained in [12], is plotted in Fig. 1. It is clear from the figure that most merger related shocks have Mach number around $\sim 1.5$. As a consequence, the spectra of particles accelerated in most merger related shocks 


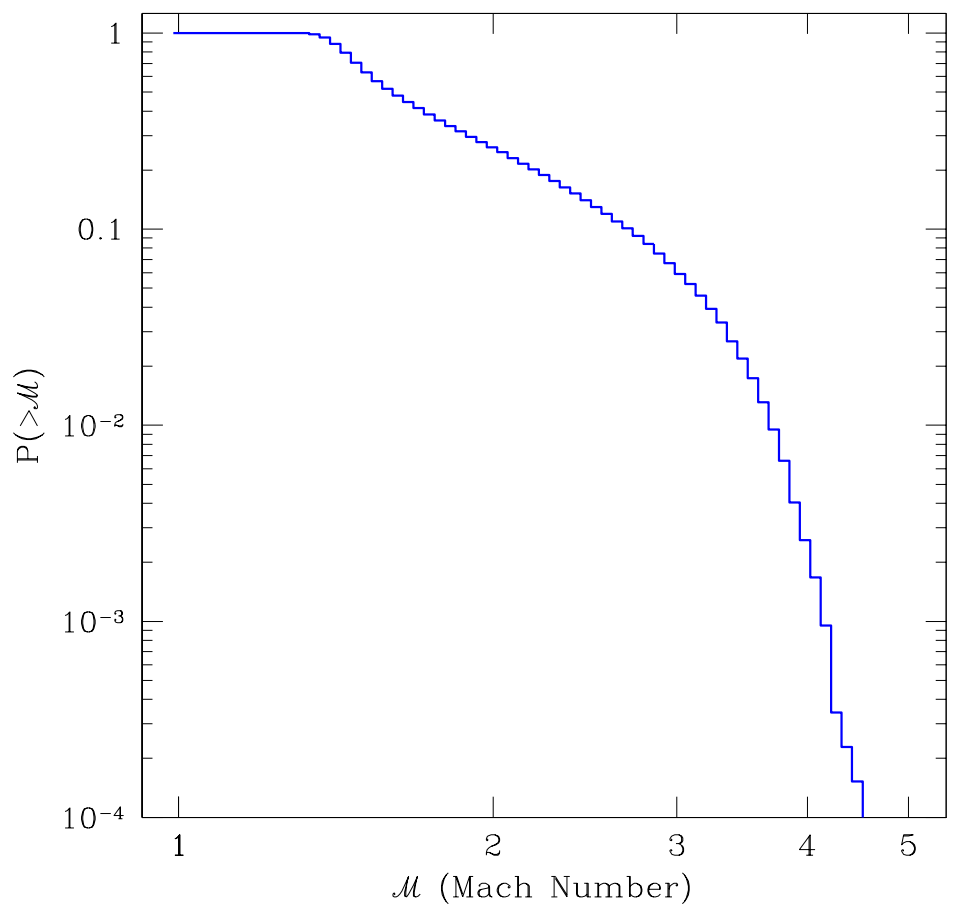

Fig. 1. Cumulative distribution of Mach numbers in mergers of clusters of galaxies.

are predicted to be extremely steep, and therefore of marginal importance from the point of view of explaining the observed nonthermal phenomena in clusters of galaxies. On the other hand, the strong shocks expected in the case of mergers between clusters with very different masses may generate periods of large gamma ray emission lasting approximately for the same duration of the merger itself. This is expected to happen in $\sim 5 \%$ of the cases, as derived from Fig. 1 [12].

During the mergers, a population of newly accelerated protons is also injected in the intracluster medium. These protons can contribute high energy gamma rays through the decay of neutral pions and high energy electrons through the decay of charged pions [3,29,8,26-28]. As pointed out in [13], an hadronic feature might appear on top of the gamma ray emission generated by ICS of electrons, even if protons are accelerated with the same efficiency as electrons.

However it is important to keep in mind that the proton component cannot escape the cluster volume, therefore the energy density in accelerated protons in a cluster depends wildly upon the merger history of the cluster, and on the microphysics of the shock acceleration process, as will be discussed in a forthcoming paper. This is mainly a consequence of the re-energization of the protons stored in the intracluster medium each time that a new merger event takes place. This problem deserves a detailed discussion per se, and we decided to dedicate a separate paper to this issue. 
It may be useful to summarize the content of this section as follows: structure formation occurs through hierarchical clustering, consisting of mergers of smaller clusters, which provide the largest contribution to the mass enhancement, and of accretion of matter onto the evolving potential well. Both processes are characterized by the formation of shocks with very different properties: merger shocks are mostly weak and occur in the central parts of clusters, while accretion shocks form in the outskirts and have large Mach numbers. The results about merger shocks, obtained in [12] were initially discrepant to the results of numerical simulations [19], which found a bimodal distribution of Mach numbers with two peaks at Mach numbers $\sim 5$ and 1000. More recent numerical simulations [14] found that the peak at lower Mach number is in fact at 1.5 as previously obtained with our semi-analytical calculations [12]. The peak at larger Mach numbers in [14] is simply due to accretion shocks. These shocks were not included in our initial paper [12] and were introduced later in a separate pubblication [9].

\section{Detectability of gamma rays from clusters of galaxies with space- borne gamma ray telescopes}

As discussed in many previous papers, gamma rays can be generated in clusters of galaxies mainly through the following physical processes (see e.g. [28]):

1. ICS of electrons accelerated at merger and accretion shocks

2. ICS of secondary electrons from the decay of charged pions produced in inelastic $p p$ collisions

3. Decay of neutral pions produced in inelastic $p p$ collisions

4. Bremsstrahlung emission of relativistic primary and secondary electrons.

Due to cosmic ray confinement, protons are stored in the intracluster medium for cosmological times, and in principle can produce secondary products continuously [3], namely with no time correlation with phenomena like mergers of two clusters. While this process is of great importance and can be responsible for appreciable gamma ray emission, it is also quite uncertain because the amount of protons diffusively stored in the intracluster medium is determined by the history of the cluster. In the following we discuss only three of the elements that contribute to this uncertainty:

\section{Mergers as accelerators}

Different clusters have different merger histories, namely different merger trees. As stressed above, most mergers produce weak shocks, unable to efficiently energize cosmic ray particles. Depending on the cluster, there may be several strong shocks formed when mergers between clusters with very different masses 
take place. The energy density in protons trapped within the cluster that will eventually result from several merger events occurring during its history depends on the number and strength of the shocks that accelerated those protons.

\section{Mergers as re-accelerators}

While most electrons accelerated during a merger lose most of their energy before the next merger event, protons are simply stored in the intracluster medium and each new merger related shock re-energizes the particles trapped until that time, besides accelerating new particles. Again, the merger history affects this component in a crucial way. Moreover, the spectrum of the reenergized particles depends on the spectrum of the protons accelerated and re-energized by previous mergers. The spectrum of the re-accelerated particles also depends upon the minimum energy that the protons possess. These effects may change the spectrum of the accelerated particles trapped in the intracluster medium even by orders of magnitude, in particular at the highest energies, where the spectrum depends crucially on the amount of energy that crossed the few strong shocks developed during the merger history of the cluster. A corresponding uncertainty affects the secondary products of cosmic ray interactions in the intracluster medium, namely gamma rays and electrons (positrons).

\section{Additional sources}

The storage properties that clusters of galaxies exhibit for the proton component make them very sensitive to all nonthermal events occurring within the cluster. In particular, acceleration processes in radio galaxies in cluster cores pollute the intracluster medium with accelerated protons which have no connection with shocks related to structure formation.

All these uncertainties can be seen as pieces of physics of the cosmic ray acceleration in clusters of galaxies that require further investigation, and will be discussed at length in a forthcoming publication. In the present paper on the other hand we adopt a conservative attitude and restrict our attention to the gamma ray emission that is solely the result of ICS of relativistic electrons, accelerated at merger and accretion shocks, against photons of the CMB radiation. Any contribution to the gamma ray emission from proton interactions can only increase the gamma ray fluxes derived below.

We start our discussion from accretion shocks, whose role is simpler to investigate since their Mach number is extremely large and the spectrum of the particles they accelerate is almost exactly a power law $E^{-2}$. The balance between energy losses, dominated by ICS, and continuous injection of newly accelerated electrons, drives the injection spectrum towards an equilibrium spectrum that is one power steeper than the injection. 
The energy per unit time converted at the shock into nonthermal electrons can be written as

$$
L_{e}^{a c c}=\eta \frac{1}{2} \varrho_{b}(1+z)^{3} v_{f f}^{3} 4 \pi R_{v}^{2} \propto M^{5 / 3}
$$

where $\eta_{e}$ is the shock acceleration efficiency for electrons, fixed here at $5 \%$, $v_{f f}=\left(2 G M / R_{v}\right)^{1 / 2}$ is the free fall velocity of the gas at the virial radius $R_{v}$ and $\varrho_{b}=\Omega_{b} \rho_{c r}$ is the present mean baryonic density of the universe, if $\Omega_{b}=0.02 h^{2}$ is the baryon fraction and $\rho_{c r}$ is the critical density.

It is important to keep in mind that the accretion of gas onto the cluster only contributes a small fraction $(\sim 10 \%)$ of the total final mass, which is instead mainly built up through mergers with other massive clusters.

The differential gamma ray luminosity, expressed in photons per unit energy per unit time, is easily calculated as ICS of the CMB photons, and is proportional to $L_{e}^{a c c}$ given above. It follows that, for a given redshift, the gamma ray luminosity is a growing function of the cluster mass, so that the number of accreting objects observable by a gamma ray telescope with sensitivity $F_{l i m}$ can be written as follows:

$$
N_{a c c}\left(F_{l i m}\right)=\int_{0}^{\infty} d z \frac{d V}{d z} \int_{M\left(F_{l i m}, z\right)}^{\infty} d M n(M, z)
$$

where $d V$ is the comoving volume in the redshift region between $z$ and $z+d z$, $n(M, z)$ is given by Eq. 6 and $M\left(F_{\text {lim }}, z\right)$ is the mass of a cluster accreting at redshift $z$ whose flux is $F_{\text {lim }}$.

The case of merger shocks is slightly more complicated, due to the fact that the shock strength is a function of the masses of the clusters involved in the merger, and more specifically of the ratio of the masses. As discussed above, merger shocks are often weak, following the distribution given in Fig. 1. Flat electron spectra, necessary to generate an appreciable gamma ray emission above $100 \mathrm{MeV}$, are obtained at strong shocks that form only during mergers between clusters with very different masses. In the approach presented in [9], at each merger events two shocks are generated, each propagating in one of the merging clusters. The Mach numbers of both shocks can be calculated from the relative velocity of the clusters and if the sound speed in each cluster is known. The slopes of the spectra of the accelerated electrons easily follows as $\alpha=2\left(\mathcal{M}^{2}+1\right) /\left(\mathcal{M}^{2}-1\right)$, where $\mathcal{M}$ is the relevant Mach number. 
The rate at which energy is channelled into nonthermal electrons in merger related shocks can be evaluated as

$$
L_{e}^{m e r}=\eta \frac{1}{2} \varrho_{b, i} v^{3} S_{i}
$$

where $\varrho_{b, i}$ is the baryonic density of the i-th cluster, $S_{i}$ is the surface of the shock that propagates in the i-th cluster and $v$ is the merger relative velocity. The gamma ray luminosity due to ICS of these accelerated electrons is calculated as usual.

The number of observable merging clusters is:

$$
\begin{gathered}
N_{\text {mer }}\left(F_{\text {lim }}\right)=\int_{0}^{\infty} d z \frac{d V}{d z} \int_{M_{\text {min }}}^{\infty} d M_{1} n\left(M_{1}, z\right) \times \\
\int_{M_{\text {min }}}^{M_{1}} d M_{2} \mathcal{R}\left(M_{1}, M_{1}+M_{2}, z\right) \Delta t_{m e r} \vartheta\left[F_{l i m}-F_{\gamma}\left(M_{1}, M_{2}, z\right)\right],
\end{gathered}
$$

where $F_{\text {lim }}$ is the telescope sensitivity, $F_{\gamma}\left(M_{1}, M_{2}, z\right)$ is the gamma ray flux we receive when a cluster with mass $M_{1}$ merges with a cluster with mass $M_{2}$ at a redshift $z, \Delta t_{\text {mer }}=R_{2} / v$ is the duration of the merger event, $M_{\text {min }}=10^{13} M_{\odot}$ is the typical mass for galaxy groups and $\vartheta$ is the Heaviside step function. The aim of this section is to determine the $\log N-\log S$ distribution of clusters of galaxies as gamma ray sources, for the cases of mergers and accretion. The results of our calculations are shown in Fig. 2 in the form of number of clusters with gamma ray emission at energies in excess of $100 \mathrm{MeV}$ larger than some value $F$ on the x-axis: the solid and dashed lines refer to accretion and mergers respectively. The vertical lines correspond to the GLAST, AGILE and EGRET sensitivities for point sources, as indicated in the plot.

The fact that the two curves in Fig. 2 overlap almost exactly is the result of a combination of many factors: each merger is on average much more luminous that accretion, because the energy involved is larger and the time during which it is released is shorter $\left(\sim 10^{9}\right.$ years for a merger versus the cluster lifetime for accretion). On the other hand, most of the times the merger event does not result into gamma ray emission, because the shocks involved are too weak. The slight deviation from a power law at low fluxes in Fig. 2 is the consequence of the cosmological effects in a non-euclidean geometry. The change of slope appears at larger fluxes for the mergers (dashed line) than for accretion. This is a result of the larger luminosity per merger that allows one to detect a bright merger from larger distances but also the consequence of the strong evolution in the merger luminosity. This latter effect is easy to explain: for a minimum cluster mass of $\sim 10^{13} M_{\odot}$, the flat spectra are obtained only when a merger occurs with a cluster of mass $\sim 10^{15} M_{\odot}$; these clusters are already on the tail 


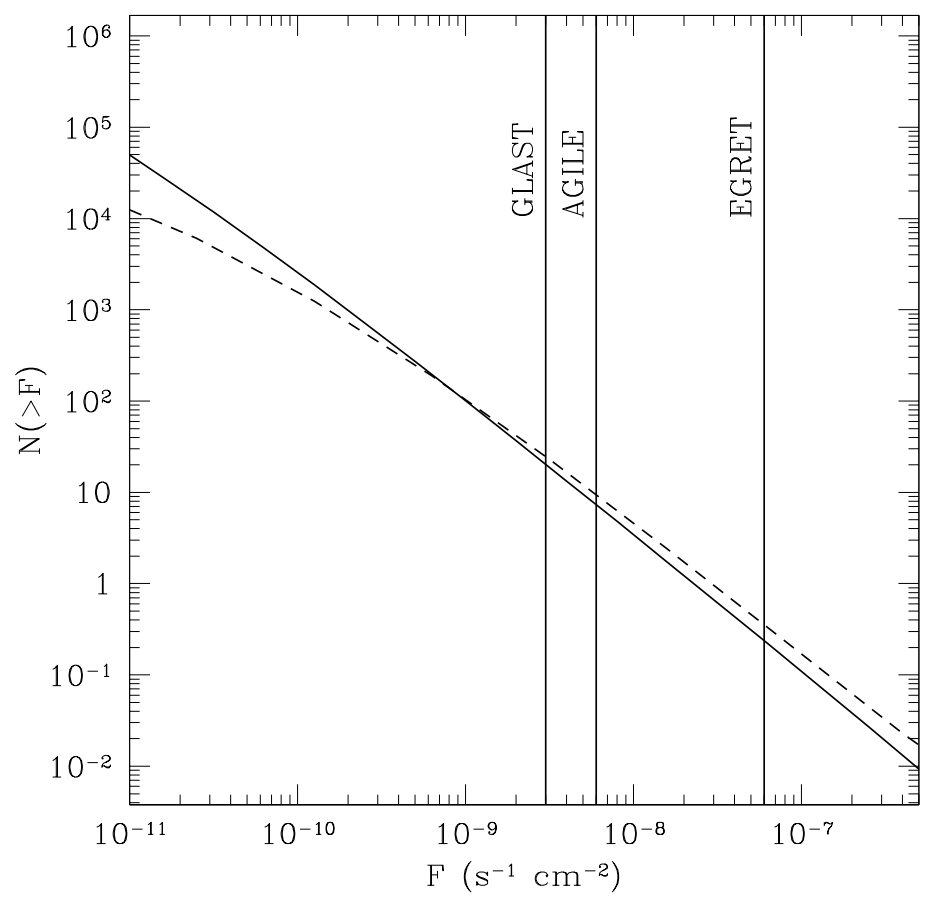

Fig. 2. Number of accreting (solid line) and merging (dashed line) clusters with gamma ray flux greater than $F$. The vertical lines represent the GLAST, AGILE and EGRET sensitivity for point sources.

of the PS distribution at the present cosmic time, while they had not been formed yet at much earlier times. Despite the larger luminosity of the mergers (on average), it is interesting to notice that the gamma ray emission for the merger case is limited to the merger period or shortly after, while the gamma ray emission associated to accretion is continuous, although less intense.

Our calculations show that $\sim 50$ clusters should be detected in gamma rays above $100 \mathrm{MeV}$ by GLAST, equally distributed between merging and accreting clusters. AGILE on the other hand should be able to detect $\sim 10-20$ objects. Moreover, we predict that no cluster should have been detected by EGRET, despite the recent claims of temptative association of some unidentified EGRET sources with the location of Abell clusters [30,31].

The results summarized in Fig. 2 are compared with the sensitivity of EGRET, GLAST and AGILE as obtained for point sources. This seems justified at least for gamma rays with energy around $100 \mathrm{MeV}$ : the gamma ray emission region in clusters is approximately a few degrees wide and the point spread function of GLAST at $100 \mathrm{MeV}$ is $\sim 4^{\circ}$, while it reduces to $0.1^{\circ}$ at $10 \mathrm{GeV}$ [32]. Similar arguments hold even for the AGILE angular resolution [33].

Since our results greatly differ from some previous results, some comments and comparisons are required. In [34,35] a Press-Schechter based method similar 
to the one presented in [9] was developed in order to make predictions about the number of clusters detectable in gamma rays with energy above $100 \mathrm{MeV}$ with future gamma ray telescopes. The authors claimed that at least a few tens of clusters should have been visible to EGRET. GLAST, on the other hand, was predicted to be able to detect more than a few thousands of such objects. This prediction seemed to be supported by some preliminary observational evidences for a temptative association between unidentified high latitude EGRET sources and Abell clusters (or cluster pairs) [30,31]. The statistical significance and physical plausibility of such an association was strongly questioned in [15], in which the important conclusion was reached that we still have to await the first observational evidence for the high-energy gamma-ray emission of galaxy clusters. The lack of association between unidentified EGRET sources and clusters of galaxies was also found in [36]. These findings seem to be perfectly in line with the predictions described in the present paper.

In [34], all shocks were assumed to be strong. As a consequence, the spectrum of the accelerated particles was always taken to be $\propto E^{-2}$. As discussed above, and as described in greater details in [12,9], for merger related shocks this assumption leads to incorrect results. The gamma ray emission in [34] is overestimated by orders of magnitude (despite the fact that the fraction of the energy crossing the shock which is converted to nonthermal electrons is assumed to be the same as here, namely $5 \%$ ), and its spectrum does not reflect the real strength of the shocks developed during mergers of clusters of galaxies. This concept can be rephrased in another way: at fixed total energy flux crossing the merger shocks, and at fixed efficiency of electron acceleration, the nonthermal energy is distributed among accelerated particles in a different way if the spectrum of nonthermal electrons is taken to be $E^{-2}$ rather than as the one derived from the correct evaluation of the shocks strength. The clusters that we predict to be detectable with AGILE and GLAST in gamma rays above $100 \mathrm{MeV}$ all have flat spectra, which means that the gamma ray emission selects the few mergers that happen to have a flat spectum of accelerated electrons (note that for Mach numbers in the range 1.5 - 4, the slope of the electron spectrum spans the range $\alpha \sim 2-5$ ), in addition to the less luminous nearby accreting clusters that always generate flat spectra.

Similar arguments apply to the calculations in Ref. [35]: the formation of a cluster was exemplified there as a spherical collapse, in which $\sim 5 \%$ of the kinetic energy crossing the accretion shock was converted into nonthermal electrons with spectrum $E^{-2}$. From the energetic point of view, this approach is roughly equivalent to that of [34]. However, as discussed above, only a relatively small fraction of the mass in a cluster is expected to be accumulated through accretion, being mergers with other clusters the main physical process for mass build-up. As stressed above, the spectrum of the particles that the energy is channelled into is the key point in understanding whether one can expect appreciable gamma ray fluxes from clusters of galaxies. 


\section{Detectability of clusters with ground based Cherenkov telescopes}

In $\S 2$ we showed that if electrons are accelerated diffusively across merger and accretion shocks following Böhm diffusion, their maximum energy can be as high as several tens of $\mathrm{TeV}$, and the $\mathrm{CMB}$ photons can be upscattered through ICS to gamma ray energies up to several $\mathrm{TeV}$ if the magnetic field in the vicinity of the shock is in the range $0.1-1 \mu G$.

In this section we address the issue of detectability of the gamma rays with energy above $100 \mathrm{GeV}$ from clusters of galaxies with Cherenkov ground based detectors. The calculations of the gamma ray emission for merging and accreting clusters have already been described in the previous section. The only new ingredient that needs to be included here is the absorption of high energy gamma rays due to pair production in the universal photon background, in particular the infrared background (IRB). Pair production starts to be important when the energy in the center of mass of a photon-photon scattering equals twice the electron mass. The net result of the propagation of high energy gamma rays with energy in excess of a few hundred $\mathrm{GeV}$ is an absorption cutoff for sources more distant that the pathlength for pair production. The corresponding optical depth at energy $E$ for a source at distance $D, \tau(E, D)$, has been calculated in [37] where the authors adopt an empirical model for the IRB, poorly measured in the energy range of interest. The gamma ray flux calculated from one cluster in the previous section can be translated into a flux at the detector simply multiplying it by $\exp [-\tau(E, D)]$.

In Fig. 3 we plot the results of our calculations for the high energy gamma ray spectrum generated from a Coma-like cluster of galaxies at $100 \mathrm{Mpc}$ distance for the case of merger and accretion. The effect of the gamma ray absorption in the IRB is illustrated by the difference between the solid lines (with absorption) and dashed lines (without absorption).

From top to bottom, the lines refer to three different cases: 1) a merger between two clusters with masses $10^{15} M_{\odot}$ and $\left.10^{13} M_{\odot} ; 2\right)$ an accreting cluster with mass $10^{15} M_{\odot}$ with a magnetic field at the shock in the upstream region $0.1 \mu G$; 3) an accreting cluster with mass $10^{15} M_{\odot}$ with a magnetic field at the shock in the upstream region $0.01 \mu G$.

The thick solid lines represent the sensitivities for a generic Cherenkov telescope as calculated in [38]. These results are obtained considering an array of imaging atmospheric Cherenkov telescopes (IACT) consisting of $n$ cells, each consisting of a $100 \times 100 \mathrm{~m}^{2}$ quadrangle with four ' $100 \mathrm{GeV}$ ' class IACTs in its corners. The two thick curves in Fig. 3 represent the minimum detectable fluxes for point sources (lower curve) and an extended $1^{\circ}$ wide source (upper curve) for an exposure time of 1000 hours. The exposure time here is defined 


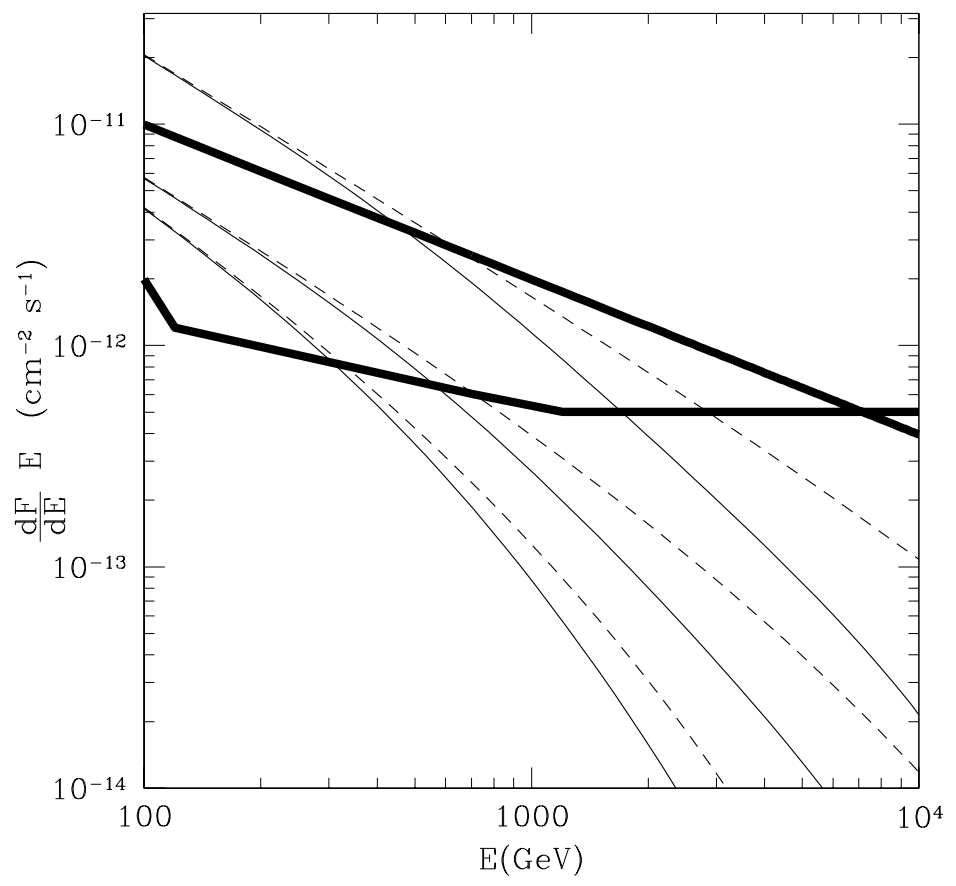

Fig. 3. Gamma ray emission in the $100 \mathrm{GeV}-10 \mathrm{TeV}$ region. The thick solid lines represent the sensitivities of a IACT for point sources (lower curve) and extended sources (upper curve). The predicted gamma ray fluxes from a Coma-like cluster at a distance of $100 \mathrm{Mpc}$ with and without absorption of the infrared background are plotted as dashed and solid lines respectively.

as the product between the observation time and the number of cells that form the array. For instance, an exposure of 1000 hours can be achieved with a 100 hours observation performed by an array consisting of 10 cells. If the angular resolution of the Cherenkov experiment is taken to be 0.1 degrees, most clusters of galaxies, both merging and accreting, are diffuse sources for these experiments. The angular size of a cluster at distance $D$ and with an emitting region of size $R=1 \mathrm{Mpc}$ is $\alpha \approx 1^{\circ}(D / 100 \mathrm{Mpc})$. For accretion, the shock surface at which acceleration occurs is located approximately at the virial radius, that for a Coma-like cluster is $\sim 3 \mathrm{Mpc}$, therefore the emission is even more extended than for a merger, and with angular size that approaches or even exceeds the aperture of the Cherenkov telescopes (a few degrees).

For the optimistic conditions that Fig. 3 refers to, the predicted flux of gamma rays between $100 \mathrm{GeV}$ and a few hundred $\mathrm{GeV}$ for a merger between two clusters with mass ratio $10^{-2}$ is only slightly above the sensitivity of a Cherenkov telescope for a 1000 hours exposure. The predicted fluxes from accretion are unobservable. The sensitivities of the telescopes might be improved to some extent, compared with the curves in Fig. 3 by simulating off-center showers and different triggering modes of the telescopes in a cell. It appears however that the perspectives for detection of $\mathrm{TeV}$ gamma radiation from ICS of ul- 
trarelativistic electrons in clusters of galaxies are not very promising.

\section{Discussion and Conclusions}

The detection of gamma ray emission from clusters of galaxies represents one of the major goals of future gamma ray telescopes. In this paper we addressed the issue of detectability of the gamma ray signal as expected from mergers of clusters of galaxies and from the so-called secondary infall of gas onto a cluster potential well. We stressed that the two phenomena are intrinsically different both for the energy involved and for the spectra of the particles accelerated at the related shock surfaces.

Gamma rays can be produced mainly due to two physical processes, namely generation and decay of neutral pions in inelastic $p-p$ collisions, and inverse Compton scattering of relativistic electrons. In this paper we concentrated our attention on the latter process, while the full account of the gamma ray emission from hadronic processes will be discussed in detail in a forthcoming paper. The reason for separating the two calculations is the peculiar behaviour of protons (or more generally nuclei) that are stored in the intracluster medium for cosmological times, causing the radiation produced at any fixed time to be the result of the full history of the cluster. The situation is made more difficult by the fact that shocks related to structure formation re-energize the protons stored in the intracluster medium, besides accelerating new cosmic rays from the thermal plasma. All these processes depend on the specific history of the cluster, determined by the merger tree that the custer experiences. Each shock associated to different mergers has different strength, therefore even the spectrum of the accelerated and re-accelerated particles depends on the merger history. As a result, the energy density in cosmic rays in a cluster may change wildly from cluster to cluster.

The situation is simpler for electrons accelerated during structure formation: the electrons responsible for the gamma ray emission through inverse Compton upscattering of the CMB photons have energies in the range $200 \mathrm{GeV}-50 \mathrm{TeV}$, whose loss time scale is between thirty thousand years and a few million years, much shorter than the time between two merger events. Most electrons are therefore simply accelerated during a merger and lost to lower energies afterwards.

Clusters of galaxies grow mainly through mergers, but secondary infall of gas onto a cluster also contributes to the mass increase, although in a smoother way, without the billion-years-long bursts that are typical of mergers.

The main differences between the gamma rays generated by particles acceler- 
ated at mergers and accretion shocks are the energy available and the spectra of accelerated particles. As found in [12] and summarized here, merger related shocks are mostly weak, their Mach number distribution having a peak at $M \sim 1.5$. These shocks generate very steep electron spectra, irrelevant from the point of view of high energy phenomena related to electrons. The only mergers able to produce flat spectra are the so-called minor mergers, consisting of the encounter of two clusters with mass ratio $\sim 0.01$. The gamma ray emission in these events is restricted to the duration of the merger and likely limited to the shock region.

The accretion shocks are located in the outskirts of clusters, at a position comparable with the virial distance from the cluster center. The energy flow per unit time across accretion shocks is smaller than that related to mergers, but it occurs continuously in time. Since these shocks carry the information about the virialization of the inner regions towards the cold outer parts of the cluster, by definition their Mach number can be very high, $M \sim 10-1000$. As a consequence, the spectrum of the particles accelerated at these shocks all have spectrum $E^{-2}$ (note that the equilibrium spectrum, due to the balance between injection and losses is one power steeper than the injection spectrum).

We calculated the $\log N-\log S$ distribution of merging and accreting clusters of galaxies and compared the expected fluxes with the sensitivities of EGRET, AGILE and GLAST. We found that no cluster was expected to be detected by EGRET, confirming a recent analysis of the old EGRET data from the direction of several Abell clusters, for which only upper limits on the gamma ray emission could be inferred [15]. Our calculation strongly disagrees with previous results found in $[34,35]$, for the reasons explained in $\S 4$.

The perspectives for detection appear to be more promising for future spaceborne gamma ray telescopes, such as AGILE and GLAST. Our calculations show that $\sim 10-20$ clusters should be detected by AGILE and $\sim 50$ clusters should appear in GLAST data, equally shared between merging and accreting clusters if an efficiency of $\sim 5 \%$ can be achieved in accelerating electrons at the shocks.

In principle, the gamma ray emission due to ICS of ultrarelativistic electrons can extend to a few tens of $\mathrm{TeV}$, so that clusters might be seen in future ground based Cherenkov experiments such as VERITAS and HESS. Unfortunately, only nearby $(\sim 100 \mathrm{Mpc})$ merging clusters of galaxies are expected to be detectable, while the detection of accreting clusters appears more problematic. 


\section{Acknowledgments}

We thank Gianfranco Brunetti for many useful discussions and ongoing collaboration. We are also grateful to Felix Aharonian for constructive correspondence about Cherenkov experiments and to Tom Jones and Dongsu Ryu for updating us on their work. This work was partially supported through grant COFIN 2002 at the Arcetri Astrophysical Observatory.

\section{References}

[1] L. Feretti The Universe at Low Radio Frequencies, Proceedings of IAU Symposium 199, held 30 Nov - 4 Dec 1999, Pune, India. Edited by A. Pramesh Rao, G. Swarup, and Gopal-Krishna, 2002., p.133

[2] R. Fusco-Femiano, D. Dal Fiume, L. Feretti, G. Giovannini, P. Grandi, G. Matt, S. Molendi and A. Santangelo, Astrophys. J. 513 (1999) L21.

[3] V. S. Berezinsky, P. Blasi, and V. S. Ptuskin, Astrophys. J. 487 (1997) 529.

[4] H. J. Völk, F. A. Aharonian, and D. Breitschwerdt, Space Sci. Rev. 75 (1996) 279 .

[5] A. Loeb, and E. Waxman, Nature 405 (2000) 156.

[6] P. Sreekumar, et al., Astrophys. J. 494 (1998) 523.

[7] A. Dar, and A. De Rújula, MNRAS 323 (2001) 391.

[8] S. Colafrancesco, and P. Blasi, Astropart. Phys. 9 (1998) 227.

[9] S. Gabici, and P. Blasi, Astropart. Phys. in press (astro-ph/0211573).

[10] U. Keshet, E. Waxman, A. Loeb, V. Springel, and L. Hernquist, Astrophys. J. 585 (2003) 128.

[11] F. Miniati, MNRAS 337 (2002) 199.

[12] S. Gabici, and P. Blasi, Astrophys. J. 583 (2003) 695.

[13] R. C. Berrington, \& C. D. Dermer, Astrophys. J. (2003) in press (astroph/0209436).

[14] D. Ryu, H. Kang, E. Hallman, and T. W. Jones, Astrophys. J. in press (astro$\mathrm{ph} / 0305164)$.

[15] O. Reimer, M. Pohl, P. Sreekumar, and J. R. Mattox, Astrophys. J. 588 (2003) 155.

[16] P. O. Lagage, and C. J. Cesarsky, Astron. \& Astrophys. 118 (1983) 223. 
[17] P. P. Kronberg, H. Lesch, and U. Hopp, Astrophys. J. 511 (1999) 56.

[18] P. Blasi, S. Burles, and A. V. Olinto, Astrophys. J. 514 (1999) L79.

[19] F. Miniati, D. Ryu, H. Kang, T.W. Jones, R. Cen, and J.P. Ostriker, Astrophys. J. $542(2000) 608$.

[20] E. Bertschinger, Astrophys. J. Suppl. 58 (1985) 39.

[21] W. H. Press, and P. Schechter, Astrophys. J. 187 (1974) 425.

[22] C. Lacey, and S. Cole, MNRAS 262 (1993) 627.

[23] T. Kitayama, PHD thesis (1997).

[24] T. T. Nakamura, and Y. Suto, Prog. Theo. Phys. 97 (1997) 49.

[25] O. Lahav, M. Rees, P. B. Lilje, and J. R. Primack, MNRAS 251 (1991) 128.

[26] P. Blasi, and S. Colafrancesco, Astropart. Phys. 122 (1999) 169.

[27] P. Blasi, Astrophys. J. 525 (1999) 603.

[28] P. Blasi, Astrophart. Phys. 15 (2001) 223.

[29] T.A. Ensslin, P.L. Biermann, P.P. Kronberg, and X.-P. Wu, Astrophys.J. 477 (1997) 560.

[30] S. Colafrancesco, Astron. \& Astrophys. 396 (2002) 31.

[31] W. Kawasaky, and T. Totani, Astrophys. J. 576 (2002) 679.

[32] See http://www-glast.slac.stanford.edu/software/IS/glast_lat_performance.htm

[33] See http://agile.mi.iasf.cnr.it/Homepage/performances_3.shtml

[34] T. Totani, and T. Kitayama, Astrophys. J. 545 (2000) 572.

[35] E. Waxman, and A. Loeb, Astrophys. J. Lett. 545 (2000) L11

[36] C. A. Scharf, and R. Mukherjee, Astrophys. J. 580 (2002) 154

[37] O. C. de Jager, and F. W. Stecker, Astrophys. J. 566 (2002) 738.

[38] F. A. Aharonian, W. Hofmann, A. K. Konopelko, and H. J. Voelk, Astropart. Phys. 6 (1997) 369. 